\title{
Thioredoxin-Glutathione and Lipoamide Systems Act as A Backup in Supporting Ribonucleotide Reductase Catalysis in Absence of Thioredoxin Reductase
}

\author{
Ajanta Chatterji \\ Amity University Kolkata \\ Kumar Sachin \\ RAJIB SENGUPTA ( $\square$ rsengupta@kol.amity.edu ) \\ Amity University https://orcid.org/0000-0002-9665-8784
}

Swami Rama Himalayan University Faculty of Medicine: Himalayan Institute of Medical Sciences

\section{Short Report}

Keywords:

Posted Date: January 17th, 2022

DOI: https://doi.org/10.21203/rs.3.rs-1212518/v1

License: (c) (1) This work is licensed under a Creative Commons Attribution 4.0 International License.

Read Full License 


\section{Abstract}

Ribonucleotide reductase (RNR) catalyzes the rate-limiting step in the conversion of ribonucleotides (NTPs) to deoxyribonucleotides (dNTPs) for the nucleotide biosynthesis. The thioredoxin system have been widely identified as hydrogen group donor for RNR catalysis. Herein, we report that glutathione (GSH) acts as a backup in supporting RNR catalysis via the Trx-GSH system in absence of TrxR. Our data also shows that the lipoamide system is capable of sustaining RNR activity in the complete absence of either Trx or glutaredoxin systems. Thus, our data corroborately supports the potential redundancy between the various antioxidant systems might seemingly emerge as a novel strategy utilized by cancer cells to sustain DNA synthesis and necessitate simultaneously targeting multiple antioxidant systems as a promising therapeutic strategy.

\section{Introduction}

Recent years have witnessed a widespread redundancy between the cell's most versatile antioxidant systems such as the Thioredoxin (Trx) and glutathione (GSH)/Glutaredoxin (Grx) systems. The Trx system comprising of thioredoxin (Trx), seleno-enzyme thioredoxin reductase (TrxR), and NADPH works in concerted action where Trx upon reducing the substrate, itself gets reduced by TrxR accepting electrons from NADPH, in order to maintain the catalytic cycle [1,2]. Trx is often referred to as a lethal gene, whose genetic ablation is expensive for the cell resulting in cell death. Glutathione (GSH), being the most predominant antioxidant system in the cell with huge substrate diversity has been noted to behave as a backup within the cell in several ways, one very relevant way is by complementing the job of TrxR in its absence and preventing cellular death [3]. These widespread redundancies and overlapping substrates between Trx and GSH help in maintaining an overall reducing environment within the cell. The classical functionality of Trx underlies donating hydrogen group to ribonucleotide reductase (RNR) for catalysis and conversion of nucleotides to deoxynucleotides $[4,18,19]$. The disulfide formed at the active site (R1 subunit) of the RNR during catalysis is reduced by the C-Terminal swinging arm of the R1 subunit via accepting electrons from Trx [4,5]. Thus, to support this catalytic cycle Trx needs to remain in its reduced form during each catalytic cycle with the help of TrxR. It has been found that cancer cells over-expresses Trx and TrxR, thus inhibiting TrxR activity might show a therapeutic promise in cancer [6, 7]. However, this is not the case and the classical TrxR inhibitors ATG (aurothioglucose) and auranofin (AF) that have been extensively used in numerous studies as pharmacological inhibitors of TrxR was not successful in killing cancer cells [3]. The study conducted by Du et al., 2012 [3] showed that physiological concentration of GSH, NADPH and glutathione reductase reduced Trx-1, which was further enhanced upon addition of glutaredoxin ( $\mathrm{Grx}$ ) to the system. This irrefutably supports that survival of TrxR $1^{-1-}$ tumors were strictly dependent on a functional GSH system to reduce oxidized Trx 1 and thus TrxR $1 \%$ cells were susceptible to pharmacological GSH deprivation [7-9]. However, no such study showed to date if GSH-mediated Trx1 reduction can aid in electron donation to support RNR catalysis in absence of TrxR1. Herein, we present a first cell-free study report that GSH-mediated Trx reduction can bypass the 
need of TrxR and support RNR catalysis in absence of TrxR1. Likewise, the cytosol is endowed with other direct and indirect antioxidant systems such as the lipoic acid (LA) and lipoamide system (LAM) comprising of Lipoamide (LAM), lipoamide dehydrogenase (LD), and reducing equivalent NADH [10, 13, 14]. It is well noted that TrxR and NADPH can also reduce oxidized LAM independent of LD and NADH $[11,12]$. Here, we have found for the first time that LAM is also capable of directly reducing the disulfide formed at the catalytic subunit of RNR and support RNR activity in absence of TrxR/Trx by utilizing LD and NADH. Thus, we rationalize that these widespread redundancies between the various antioxidant systems in the cell might potentially constrain chemotherapeutic treatments and contribute to cancer resistance. Thus, our data behold on concomitantly targeting/inhibiting multiple antioxidant systems within the cell might synergize in killing tumor cells and rule out to have a promising future implication in cancer.

\section{Materials And Methods:}

\subsection{Materials and reagents}

All chemicals and reagents were purchased from Sigma-Aldrich Chemicals, unless stated otherwise. The $\left[{ }^{3} \mathrm{H}\right]$ Cytidine 5'-monophosphate was from Moravek Biochemicals and Radiochemicals. The cation exchanger resin $A G \AA 50 \mathrm{~W}$ was purchased from Bio-Rad.

\subsection{Expression and purification of RNR subunits}

Mouse R1 and R2 subunits were cloned in E.coli BL21 (DE3). The plasmids were a kind gift from Dr. Lars Thelander (Umeå University, Umeå, Sweden). These plasmids containing mouse R1 and R2 were transformed in BL21 (DE3) and the protein purification was done as described previously [15, 16, 17, 21].

\subsection{Expression and purification of recombinant mouse Trx}

Similar to the above, expression and purification of recombinant mouse Trx 1 (6x-His tagged) was performed as previously described $[15,16,17,21]$.

\subsection{Conversion of $\left[{ }^{3} \mathrm{H}\right] \mathrm{CMP}$ to $\left[{ }^{3} \mathrm{H}\right] \mathrm{CDP}$ by CMP Kinase}

E. coli CMP kinase plasmid was provided by Prof. AnneMarie Gilles (Laboratoire d'Enzymologie et de Biochimie Structurales, France). The plasmid was expressed, purified, and used to convert $\left[{ }^{3} \mathrm{H}\right] \mathrm{CMP}$ to $\left[{ }^{3} \mathrm{H}\right] \mathrm{CDP}$ as described by Bucurenci et al. [22]. The [ $\left.{ }^{3} \mathrm{H}\right] \mathrm{CDP}$ was separated from the reaction mixture by a C18 HPLC column using a mobile phase comprising $20 \mathrm{mM}$ ammonium acetate buffer, $\mathrm{pH}$ 5, and 5\% acitonitrile [21]. The obtained $\left[{ }^{3} \mathrm{H}\right]$ CDP was used in RNR activity assays.

\subsection{RNR activity assay}

Determination of RNR activities were carried out as described previously [21]. Briefly, the RNR system was reconstituted by mixing recombinant $200 \mu \mathrm{g} / \mathrm{ml} \mathrm{R} 1$ and $100 \mu \mathrm{g} / \mathrm{ml} \mathrm{R} 2$ proteins. Activity was assayed following the conversion of $\left[{ }^{3} \mathrm{H}\right] \mathrm{CDP}$ into $\left[{ }^{3} \mathrm{H}\right] \mathrm{dCDP}$. The amount of radioactivity derived from $\left[{ }^{3} \mathrm{H}\right] \mathrm{dCMP}$ 
was quantified by liquid phase scintillation counting after ion exchange chromatography on Dowex-50 columns [21].

\section{Results}

\subsection{Trx-GSH system supports RNR activity-}

The experiment was carried out using R1-R2 complex in presence of $\mathrm{Mg}^{2+}$ and ATP, followed by the addition of $\left[{ }^{3} \mathrm{H}\right] \mathrm{CDP}$ to the reaction mixture as mentioned in detail in the materials and methods. To this mixture Trx 1 was added in presence of GSH as the reducing system. It was found that physiological concentrations of GSH along with 5 or $10 \mu \mathrm{M}$ Trx was able to support RNR activity. RNR activity was measured by the nanomoles of dCDP formed after 30 mins of incubation (Figure 1). It is introspective from the data that the physiological concentration of GSH can reduce oxidized Trx and support Trx mediated RNR catalysis without the presence of TrxR in the system. From our previously reported data Sengupta et al., 2019 [21] it is noteworthy to point out that although Trx-TrxR is much more efficient in supporting RNR catalysis, Trx-GSH astonishingly supports intermediate levels of RNR catalysis presumably at higher concentrations of GSH (7-10 mM, physiologically relevant) in absence of TrxR.

\subsection{Lipoamide system directly supports RNR catalysis-}

To the RNR assay system, neither Trx nor Grx was added as electron donor which was substituted with the lipoamide system comprising of $1 \mathrm{unit} / \mathrm{ml}$ LD, $1 \mathrm{mM} \mathrm{NADH}$, and the indicated concentration of LAM. Our data showed us for the first time that LAM is also capable of reducing RNR disulfide along with LD and NADH in the system and support RNR activity without the need for Trx/Grx systems. It was observed that in presence of the LAM system, RNR activity increased in a concentration-dependent manner. Impact of different concentrations of LAM on the RNR activity was monitored as the nanomole of dCDP produced after $30 \mathrm{~min}$ of reaction (Figure 2). Our data congruently provides a first report that the LAM system supports RNR activity in absence of the Trx/Grx system to sustain nucleotide synthesis.

\section{Discussion}

We wanted to investigate if RNR activity was sustained in absence of TrxR and have performed the RNR assay with the Trx-GSH system. According to our findings, GSH can compensate for TrxR and reduce Trx 1 which in turn donates electrons to support RNR activity, thus GSH is acting as a backup in supporting RNR catalysis (Figure 3). However, as compared to the traditional Trx-TrxR system (figure 3A of ref [21]), Trx-GSH system is relatively less efficient in supporting RNR activity and is strictly GSH concentrationdependent. Nonetheless, it was seen that both Trx and GSH at physiologically relevant concentrations in the assay system supported RNR activity. This profoundly brings into notice the possible mechanism that might take place, where GSH act as a backup that keeps the RNR activity uninterrupted following TrxR inhibition or ablation and facilitate cell survival. Moreover, as GSH concentration gets enhanced in cancer cells which can also put together amenable evidence that elevated GSH plays a vital role in tumorigenesis 
$[9,20]$. We have also found for the first time that the LAM system is capable of directly reducing RNR that supported intermediate levels of RNR activity without the involvement of either Trx/Grx systems. Moderate levels of RNR activity were detected in presence of LAM which directly reduced the disulfide formed at the catalytic subunit of RNR. We rationally anticipate that cells treated with TrxR inhibitors/ TrxR-knockout utilize these backup antioxidant systems to cumulatively sustain RNR activity and cell survival that by-passes the effect of these chemo/cytotoxic treatments. Figure 3 represents a schematic model based on our study. This also reasonably supports the notion why nature has kept these antioxidant systems to be simultaneously present within the cells with widespread redundancies that could be a strategy utilized by cancer cells to turn the fate towards cell survival. Our data will open new avenues for research which could be further supported by in situ/ in vivo studies that would aid in better understanding the complexities and cross-talk between potential antioxidants, paving the route towards guiding effective treatments by concomitantly targeting multiple antioxidant systems to overcome treatment resistance.

\section{Abbreviations}

RNR, ribonucleotide reductase; GSH, glutathione; Trx, Thioredoxin; TR, Thioredoxin Reducatse; LAM, Lipoamide; LD, Lipoamide Dehydrogenase.

\section{Declarations}

Acknowledgements: This work is dedicated to the memory of Prof. Arne Holmgren (deceased January, 2020). We are grateful to Prof. Holmgren for his enormous help, support and guidance by providing resources, funding acquisition and oversight of the experiments. This work was supported by the grants from Karolinska Institutet. We gratefully thank Rolf Eliasson for excellent technical assistance, and Jacek Andrzejewski for large scale cultivation of bacteria.

Author Contribution: AC and RS wrote the manuscript. RS provided resources and funding acquisition. RS contributed to the experimental design, performed the experiments. RS and AC performed the experiments. RS, AC and KS provided critical discussion of the manuscript. RS, AC and KS reviewed the results and approved the final version of the manuscript.

Conflict of Interest Disclosure: The authors declare no competing interest.

Compliance with Ethical Standards: Not applicable.

Funding: This work was supported by the grants from Karolinska Institutet.

Ethical approval: This article does not contain any studies with animals and human participants performed by any of the authors.

\section{References}


1. Lu, J., \& Holmgren, A. (2014). The thioredoxin antioxidant system. Free radical biology \& medicine, 66, 75-87. https://doi.org/10.1016/j.freeradbiomed.2013.07.036

2. Sengupta, R., \& Holmgren, A. (2013). Thioredoxin and thioredoxin reductase in relation to reversible S-nitrosylation. Antioxidants \& redox signaling, 18(3), 259-269. https://doi.org/10.1089/ars.2012.4716

3. Du, Y., Zhang, H., Lu, J., \& Holmgren, A. (2012). Glutathione and glutaredoxin act as a backup of human thioredoxin reductase 1 to reduce thioredoxin 1 preventing cell death by aurothioglucose. The Journal of biological chemistry, 287(45), 38210-38219. https://doi.org/10.1074/jbc.M112.392225

4. Sengupta, R., \& Holmgren, A. (2014). Thioredoxin and glutaredoxin-mediated redox regulation of ribonucleotide reductase. World journal of biological chemistry, 5(1), 68-74.

https://doi.org/10.4331/wjbc.v5.i1.68

5. Eklund, H., Eriksson, M., Uhlin, U., Nordlund, P., \& Logan, D. (1997). Ribonucleotide reductase-structural studies of a radical enzyme. Biological chemistry, 378(8), 821-825.

6. Shang, W., Xie, Z., Lu, F., Fang, D., Tang, T., Bi, R., Chen, L., \& Jiang, L. (2019). Increased Thioredoxin-1 Expression Promotes Cancer Progression and Predicts Poor Prognosis in Patients with Gastric Cancer. Oxidative medicine and cellular longevity, 2019, 9291683.

https://doi.org/10.1155/2019/9291683

7. Mandal, P. K., Schneider, M., Kölle, P., Kuhlencordt, P., Förster, H., Beck, H., Bornkamm, G. W., \& Conrad, M. (2010). Loss of thioredoxin reductase 1 renders tumors highly susceptible to pharmacologic glutathione deprivation. Cancer research, 70(22), 9505-9514. https://doi.org/10.1158/00085472.CAN-10-1509

8. Bansal, A., \& Simon, M. C. (2018). Glutathione metabolism in cancer progression and treatment resistance. The Journal of cell biology, 217(7), 2291-2298. https://doi.org/10.1083/jcb.201804161

9. Traverso, N., Ricciarelli, R., Nitti, M., Marengo, B., Furfaro, A. L., Pronzato, M. A., Marinari, U. M., \& Domenicotti, C. (2013). Role of glutathione in cancer progression and chemoresistance. Oxidative medicine and cellular longevity, 2013, 972913. https://doi.org/10.1155/2013/972913.

10. Biewenga, G. P., Dorstijn, M. A., Verhagen, J. V., Haenen, G. R., \& Bast, A. (1996). Reduction of lipoic acid by lipoamide dehydrogenase. Biochemical pharmacology, 51(3), 233-238. https://doi.org/10.1016/0006-2952(95)02124-8

11. Arnér, E. S., Nordberg, J., \& Holmgren, A. (1996). Efficient reduction of lipoamide and lipoic acid by mammalian thioredoxin reductase. Biochemical and biophysical research communications, 225(1), 268-274. https://doi.org/10.1006/bbrc.1996.1165

12. Zhao, L., Liu, Z., Jia, H., Feng, Z., Liu, J., \& Li, X. (2015). Lipoamide Acts as an Indirect Antioxidant by Simultaneously Stimulating Mitochondrial Biogenesis and Phase II Antioxidant Enzyme Systems in ARPE-19 Cells. PloS one, 10(6), e0128502. https://doi.org/10.1371/journal.pone.0128502

13. Persson, H. L., Svensson, A. I., \& Brunk, U. T. (2001). Alpha-lipoic acid and alpha-lipoamide prevent oxidant-induced lysosomal rupture and apoptosis. Redox report : communications in free radical research, 6(5), 327-334. https://doi.org/10.1179/135100001101536472 
14. Hou, Y., Li, X., Peng, S., Yao, J., Bai, F., \& Fang, J. (2019). Lipoamide Ameliorates Oxidative Stress via Induction of Nrf2/ARE Signaling Pathway in PC12 Cells. Journal of agricultural and food chemistry, 67(29), 8227-8234. https://doi.org/10.1021/acs.jafc.9b02680

15. Mann GJ, Gräslund A, Ochiai E, Ingemarson R, Thelander L (1991) Purification and characterization of recombinant mouse and herpes simplex virus ribonucleotide reductase R2 subunit. Biochemistry 30(7):1939-1947. https://doi.org/10.1021/bi002 21a030

16. Davis R, Thelander M, Mann GJ, Behravan G, Soucy F, Beaulieu P, Lavallée P, Gräslund A, Thelander L (1994) Purification, characterization, and localization of subunit interaction area of recombinant mouse ribonucleotide reductase R1 subunit. J Biol Chem 269(37):23171-23176

17. Lundberg M, Johansson C, Chandra J, Enoksson M, Jacobsson G, Ljung J, Johansson M, Holmgren A. Cloning and expression of a novel human glutaredoxin (Grx2) with mitochondrial and nuclear isoforms. J Biol Chem. 2001 Jul 13;276(28):26269-75. doi: 10.1074/jbc.M011605200. Epub 2001 Apr 10. PMID: 11297543.

18. Zahedi Avval, F., \& Holmgren, A. (2009). Molecular mechanisms of thioredoxin and glutaredoxin as hydrogen donors for Mammalian s phase ribonucleotide reductase. The Journal of biological chemistry, 284(13), 8233-8240. https://doi.org/10.1074/jbc.M809338200

19. Chatterji, A., \& Sengupta, R. (2021). Cellular S-denitrosylases: Potential role and interplay of Thioredoxin, TRP14, and Glutaredoxin systems in thiol-dependent protein denitrosylation. The international journal of biochemistry \& cell biology, 131, 105904. https://doi.org/10.1016/j.biocel.2020.105904

20. Chatterji, A., Holmgren, A., \& Sengupta, R. (2021). Ribonucleotide reductase: In-vitro Sglutathionylation of R2 and p53R2 subunits of mammalian class I ribonucleotide reductase protein. Molecular biology reports, 48(11), 7621-7626. https://doi.org/10.1007/s11033-021-06721-2

21. Sengupta, R., Coppo, L., Mishra, P., \& Holmgren, A. (2019). Glutathione-glutaredoxin is an efficient electron donor system for mammalian p53R2-R1-dependent ribonucleotide reductase. The Journal of biological chemistry, 294(34), 12708-12716. https://doi.org/10.1074/jbc.RA119.008752

22. Bucurenci, N., Sakamoto, H., Briozzo, P., Palibroda, N., Serina, L., Sarfati, R. S., Labesse, G., Briand, G., Danchin, A., Bărzu, O., \& Gilles, A. M. (1996). CMP kinase from Escherichia coli is structurally related to other nucleoside monophosphate kinases. The Journal of biological chemistry, 271(5), 28562862. https://doi.org/10.1074/jbc.271.5.2856

\section{Figures}




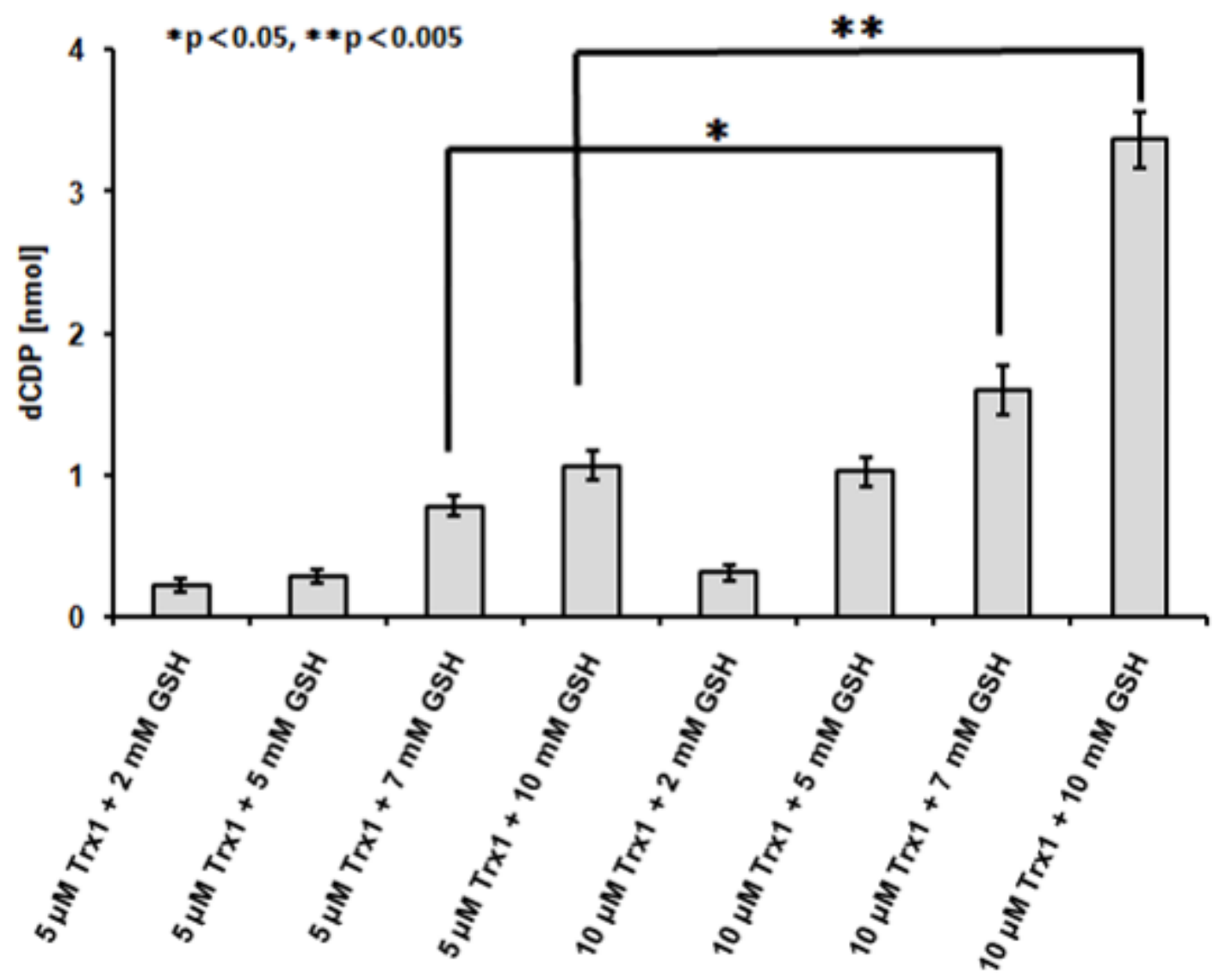

Figure 1

Trx-GSH system supports ribonucleotide reductase activity. The RNR activity was studied with reconstituted recombinant RNR system (see methods for more details) in presence of 5 or $10 \mu \mathrm{M}$ Trx 1 with the indicated concentration of GSH. RNR activity increased in a concentration dependent manner of both GSH and Trx1, where the activity was detectable at $5 \mu \mathrm{M}$ Trx and $7-10 \mathrm{mM} \mathrm{GSH}$; and was increased at $10 \mu \mathrm{M}$ Trx and 7-10 mM GSH ( $\left.{ }^{\star} P<0.05, \star \star *<<0.005\right)$. Thus, physiological concentration of Trx and GSH supports RNR activity in absence of TrxR. 


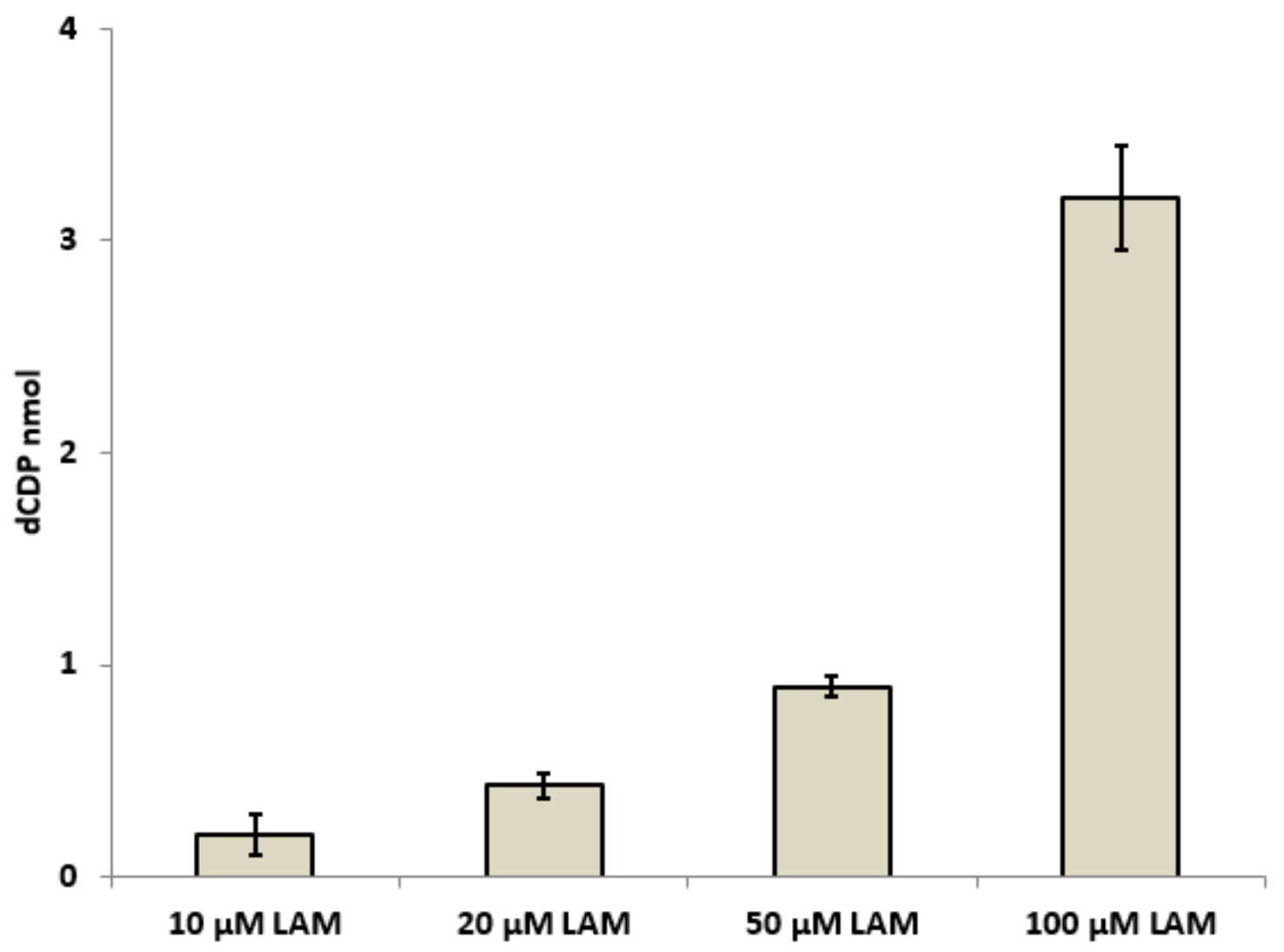

Figure 2

Lipoamide system supports ribonucleotide reductase activity. The RNR activity was studied with reconstituted recombinant RNR system (see methods for more details) in presence of $1 \mathrm{unit} / \mathrm{ml} \mathrm{LD}, 1 \mathrm{mM}$ $\mathrm{NADH}$, and indicated concentration of LAM. The $\mathrm{x}$-axis plotted lipoamide concentration in $\mu \mathrm{M}$ against $y$ axis plotted ribonucleotide reductase enzyme activity in nmol dCDP formed shows that 20-50 $\mu \mathrm{M}$ LAM supports basal level RNR activity, which drastically increases with an increase in LAM concentration to $100 \mu \mathrm{M}$. 


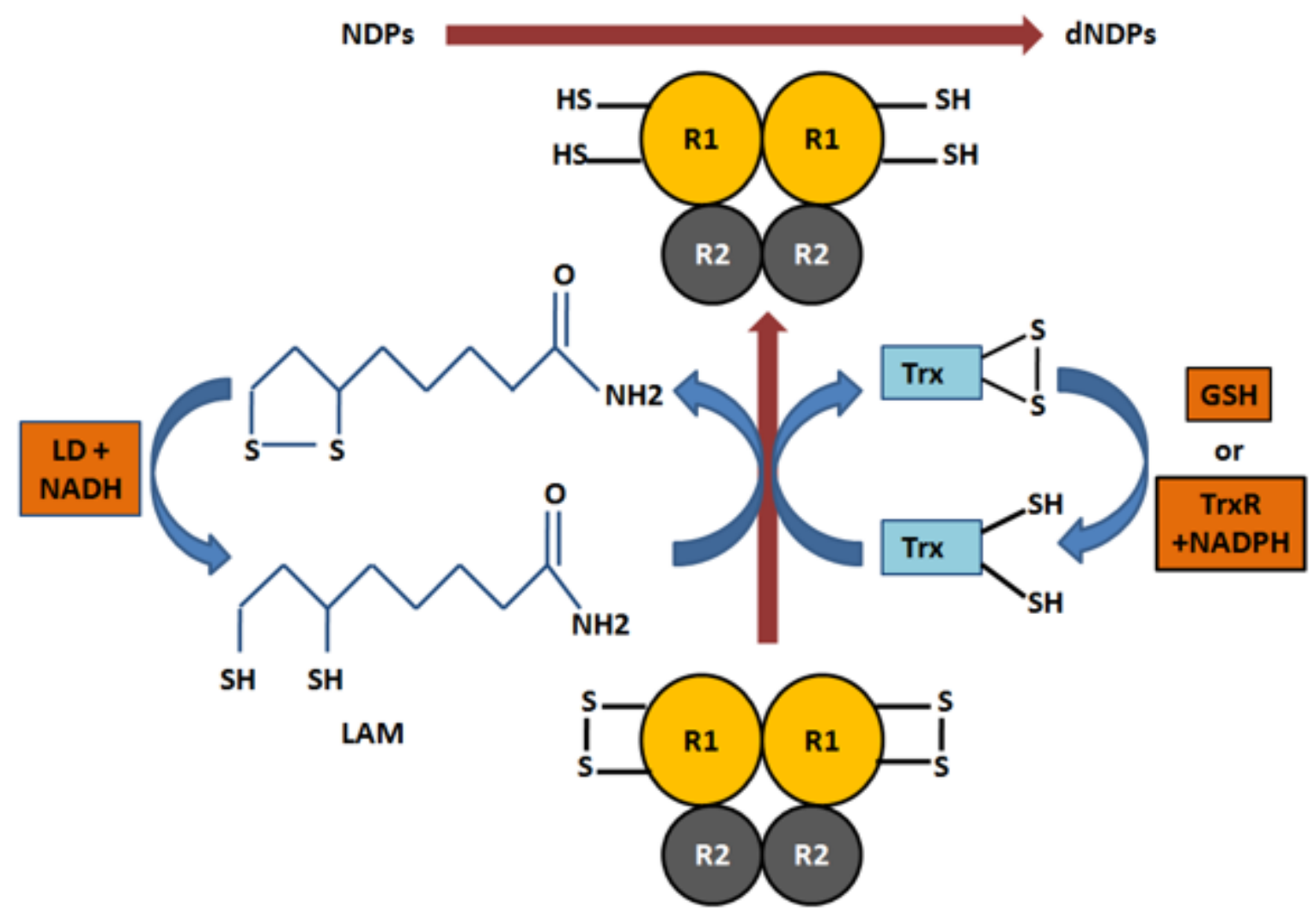

Figure 3

Model of RNR catalysis supported by Trx-GSH and Lipoamide system: We rationalize a possible mechanism that is imparted by the two systems to support RNR catalysis. There for simplicity of the figure and better understanding two disulfide bonds on R1 dimer have been shown. Left side of the figure shows Lipoamide system mediated RNR catalysis. Here, Lipoamide (LAM) directly reduces the disulfide formed at the R1 subunit of RNR resulting in formation of a disulfide in LAM. Following which it itself gets reduced by lipoamide dehydrogenase (LD) accepting electrons from NADH. This mechanism is able to support RNR activity during each catalytic cycle without the involvement of thioredoxin or glutaredoxin systems. Right side of the figure shows Thioredoxin-glutathione (Trx-GSH) mediated RNR catalysis. Here, the disulfide formed at the active site R1 subunit of RNR is reduced by C-Terminal swinging tail of R1 subunit, following accepting electrons from Trx-(SH) 2 . This results in formation of oxidized Trx (Trx-(S) $\left.{ }_{2}\right)$ which is further reduced by GSH. This mechanism points out a possible backup role of GSH system in reducing oxidized Trx, complementing the job of TrxR. We hypothesize that GSH reduces Trx, which helps in RNR catalysis in absence of TrxR. 